predict elongation at one year. A cut off of 7\% elongation at baseline would have caught $77 \%$ of patients who ended up with an elongation above $10 \%$ at 1 year.

\section{ACUTE ACHILLES TENDON RUPTURE - THE INFLUENCE OF GENDER, AGE AND COMORBIDITY ON TREATMENT OUTCOME}

${ }^{1}$ Allan Cramer*, ${ }^{1}$ Nanna Cecilie Jacobsen, ${ }^{2}$ Maria Swennergren Hansen, ${ }^{3}$ Håkon Sandholdt, ${ }^{1}$ Per Hölmich, 'Kristoffer Barfod. 'Sports Orthopedic Research Center - Copenhagen (SORC C), Arthroscopic Center, Department of Orthopedic Surgery, Copenhagen University Hospital, Amager-Hvidovre, Denmark; ${ }^{2}$ Physical Medicine and Rehabilitation Research Copenhagen (PMR-C), Denmark; ${ }^{3}$ Clinical Orthopedic Research Hvidovre (CORH), Copenhagen University Hospital, Amager-Hvidovre, Denmark

10.1136/bjsports-2019-scandinavianabs. 15

Introduction Studies suggest that women have worse treatment outcome than men after acute Achilles tendon rupture (ATR). Few studies have assessed the influence of age and comorbidity on treatment outcome after ATR. The aim of the study was to investigate if gender, age and comorbidity affect patient reported outcome following ATR.

Materials and methods The study was performed as a registry study in the Danish Achilles tendon Database. The endpoints were the Achilles tendon rupture score (ATRS) at 4 months, 1 year and 2 years after injury. Variables of interest were gender, age, diabetes, hypertension, rheumatic disease, smoking and previous Achilles tendon disorder.

Results Data were collected from April 2012 to March 2018. 1524 patients participated at 4 months, 1277 at 1 year and 899 at 2 years. Women had statistically significantly lower ATRS at 4 months (mean difference, [confidence interval], pvalue) (4.8, [1.78;7.78], $\mathrm{p}<0.01)$ and 1 year (9.9, [4.3;15.5], $\mathrm{p}<0.01$ ), but not after 2 years. Patients with hypertension (7.6, [1.4;13.8], $\mathrm{p}=0.02)$ and non-operatively treated patients with rheumatic disease $(14.8,[0.4 ; 29.2], \mathrm{p}=0.04)$ had lower ATRS at 1 year. Age showed a weak correlation to ATRS at 1 year $(\mathrm{r}=0.12 ; \mathrm{p}<0.01)$.

Conclusion Women scored statistically significantly less than men in ATRS at 4 months and 1 year after ATR. The difference was half the clinically relevant difference at 4 months and peaked at 1 year where it equaled the clinically relevant difference. Hypertension and rheumatic disease led to statistically significantly decreased ATRS. Age did not have clinical relevant influence on ATRS.

\section{RELATIONSHIPS BETWEEN A MULTIDIRECTIONAL REACTIVE AGILITY TEST, FUNCTIONAL PERFORMANCE AND PATIENT-REPORTED OUTCOME MEASURES 6 MONTHS AFTER ANTERIOR CRUCIATE LIGAMENT RECONSTRUCTION}

\footnotetext{
${ }^{1}$ Bart Dingenen*, ${ }^{2,3}$ Jan Truijen, ${ }^{2,3}$ Johan Bellemans, ${ }^{4,5,6}$ Alli Gokeler. ${ }^{1}$ Rehabilitation Research Centre, Biomedical Research Institute, Faculty of Medicine and Life Sciences, UHasselt, Agoralaan A, België; ${ }^{2}$ Department Department of Orthopedic Surgery, Ziekenhuis Oost-Limburg, Belgium; ${ }^{3}$ Faculty of Medicine and Life Sciences, UHasselt, Agoralaan, Belgium; ${ }^{4}$ Luxembourg Institute of Research for Orthopedics, Medicine and Science in Sports, Luxembourg; ${ }^{5}$ Applied Neuroscience in Sports and Exercise, Department Exercise and Health, Faculty of Science, University of Paderborn, Germany; ' University of Groningen, University Medical Center Groningen, Center for Human Movement Sciences, The Netherlands
}

10.1136/bjsports-2019-scandinavianabs. 16
Introduction Return-to-sport testing after anterior cruciate ligament (ACL) reconstruction traditionally occurs during preplanned activities. The aim of this study was to investigate the relationships between a novel multidirectional reactive agility test, functional performance and patient-reported outcome measures in athletes after ACL reconstruction.

Materials and methods Twenty-eight ACL-reconstructed athletes (24 males, 4 females; $24.6 \pm 4.4$ years; 6 months postoperative), participated in the study. All athletes underwent an evaluation including a novel multidirectional reactive agility test (tested with Smartgoals, a light-based reactive training system to measure the time to complete a task), functional performance tests: 1) two hop tests (single-leg hop for distance, triple hop for distance), 2) the Y-balance test conducted with eyes closed and patient-reported outcome measures (ACL-Return to Sports after Injury (ACL-RSI) scale, Knee Self-Efficacy Scale (K-SES), International Knee Documentation Committee (IKDC) subjective knee form). Spearman correlation coefficients were calculated between the outcomes on the multidirectional reactive agility test, and the functional performance and patientreported outcome measures.

Results The time to complete the multidirectional reactive agility test was significantly $(\mathrm{p}<0.05)$ negatively correlated with absolute hop test distances $(\mathrm{r}=-0.52$ to -0.53$)$, hop tests limb symmetry indices $(\mathrm{r}=-0.41$ to -0.49$)$, posteromedial $(\mathrm{r}=-0.64)$ and posterolateral $(\mathrm{r}=-0.61)$ reach distances on the Y-balance test, and K-SES future $(\mathrm{r}=-0.39)$, ACL-RSI $(\mathrm{r}=-0.39)$ and IKDC scores $(r=-0.44)$.

Conclusion Faster reactive agility was significantly correlated with better functional performance and patient-reported outcome measures. These results suggest to consider implementing multidirectional reactive agility testing within the continuum of the return-to-sport decision-making process in athletes after ACL reconstruction.

\section{SUBCLASSIFICATION OF RECREATIONAL RUNNERS WITH A RUNNING-RELATED INJURY BASED ON RUNNING KINEMATICS MEASURED WITH TWO-DIMENSIONAL VIDEO ANALYSIS}

${ }^{1}$ Bart Dingenen*,2Filip Staes, ' Romy Vanelderen, 'Linde Ceyssens, ${ }^{3,5}$ Peter Malliaras, ${ }^{4,5,6}$ Christian Barton, ${ }^{7}$ Kevin Deschamps. ${ }^{1}$ Rehabilitation Research Centre, Biomedical Research Institute, Faculty of Medicine and Life Sciences, UHasselt, Agoralaan A, Belgium; ${ }^{2}$ KU Leuven Musculoskeletal Rehabilitation Research Group, Department of Rehabilitation Sciences, Faculty of Kinesiology and Rehabilitation Sciences, Belgium; ${ }^{3}$ Department of Physiotherapy, School of Primary and Allied Health Care, Faculty of Medicine, Nursing and Health Science, Monash University, Australia; ${ }^{4}$ La Trobe Sport and Exercise Medicine Research Centre, School of Allied Health, La Trobe University, Australia; ${ }^{5}$ Complete Sports Care, Australia; ${ }^{6}$ Department of Surgery, St Vincent's Hospital, University of Melbourne, Australia; ${ }^{7} \mathrm{KU}$ Leuven, Department of Rehabilitation Sciences, Faculty of Kinesiology and Rehabilitation Sciences, Campus Bruges, Belgium

\subsection{6/bjsports-2019-scandinavianabs. 17}

Introduction The aim of this study was to explore whether homogeneous subgroups could be classified within the running kinematics of a group of recreational runners with a runningrelated injury (RRI).

Materials and methods Fifty-three recreational runners (15 males, 38 females) with an RRI ran on a treadmill at preferred speed. Digital videos were recorded in the frontal and sagittal plane with two iPads. Outcome measures included foot and tibia inclination at initial contact, and hip adduction and knee flexion during midstance. All angles were manually 
drawn using Kinovea and an average of seven consecutive steps was calculated for each angle. The four outcome measures were clustered using K-means cluster analysis $(n=2-10)$. Silhouette coefficients were used to detect optimal clustering. Results The cluster analysis led to the classification of two distinct subgroups (mean silhouette coefficient $=0.53$ ). Cluster 1 $(n=39)$ was characterized by higher foot inclination and tibia inclination at initial contact, higher knee flexion during midstance, and lower hip adduction during midstance compared to cluster $2(n=14)$. Fifteen out of $17(88 \%)$ shin injuries were classified in cluster 1 . Other injuries were more divided over both clusters. The ratio males/females was higher in cluster $1(44 \%)$ versus cluster $2(27 \%)$.

Conclusion This is the first study to classify subgroup profiles of running kinematics in recreational runners with an RRI based on two-dimensional video analysis. Two distinct subgroups were identified. This subclassification can help clinicians in their clinical reasoning process when evaluating kinematics of runners with an RRI and developing targeted gait-retraining strategies.

\section{TWO-DIMENSIONAL VIDEO ANALYSIS DURING RUNNING IN RECREATIONAL RUNNERS WITH AND WITHOUT RUNNING-RELATED KNEE INJURY}

\begin{abstract}
${ }^{1}$ Bart Dingenen*, ${ }^{2,3}$ Peter Malliaras, ${ }^{1}$ Tessa Janssen, ${ }^{1}$ Linde Ceyssens, ${ }^{1}$ Romy Vanelderen, ${ }^{3,4,5}$ Christian Barton. ${ }^{1}$ Rehabilitation Research Centre, Biomedical Research Institute, Faculty of Medicine and Life Sciences, UHasselt, Agoralaan A, Belgium; '2Department of Physiotherapy, School of Primary and Allied Health Care, Faculty of Medicine, Nursing and Health Science, Monash University, Australia; ${ }^{3}$ Complete Sports Care, Australia; ${ }^{5}$ Australia Department of Surgery, St Vincent's Hospital, University of Melbourne, Australia; ${ }^{4}$ La Trobe Sport and Exercise Medicine Research Centre, School of Allied Health, La Trobe University, Australia
\end{abstract}

\subsection{6/bjsports-2019-scandinavianabs.18}

Introduction The aim of this study was to compare running kinematics between recreational runners with and without running-related knee injury using two-dimensional video analysis. Materials and methods Forty-two recreational runners (18 injured, 24 non-injured) participated in the study. The injured group consisted of runners with anterior or lateral knee pain, resulting in altered running activity for at least one week. All participants ran on a treadmill at preferred speed. Digital videos were recorded in the frontal and sagittal plane with two iPads. Outcome measures included foot and tibia inclination at initial contact, and lateral trunk position, contralateral pelvic drop, femoral adduction, hip adduction, knee flexion and ankle dorsiflexion during midstance. All angles were manually drawn using Kinovea and an average of seven consecutive steps was calculated for each angle. Participant characteristics (gender, age, body weight, body length, body mass index, running volume before injury, running speed) and two-dimensional measured angles were compared between groups with independent t-tests (normally distributed) and the Mann Whitney $\mathrm{U}$ test (non-normally distributed).

Results There were no significant differences in participant characteristics between groups $(p>0.05)$. The injured group exhibited significantly more femoral adduction $(p=0.031)$ and hip adduction $(\mathrm{p}=0.004)$ during midstance, and significantly less foot inclination at initial contact $(p=0.026)$.

Conclusion Two-dimensional video analysis discriminated kinematics between runners with and without running-related knee injury. More femoral adduction and hip adduction during midstance, and less foot inclination at initial contact may provide gait retraining targets when treating runners with running-related knee injury.

\section{DO CLINICAL MEASURES RELATE TO RUNNING ASYMMETRIES IN PATIENTS WITH ACHILLES TENDINOPATHY?}

Patrick Corrigan*, Karin Grävare Silbernagel. University of Delaware, USA

10.1136/bjsports-2019-scandinavianabs.19

Introduction Achilles tendinopathy is an overuse injury that commonly sidelines runners and has a reinjury rate of $44 \%$. Aberrant loading patterns during the return-to-sports (RTS) phase may partially explain reinjury rates, but it remains unknown if clinical measures are associated with loading behaviors. Therefore, we aimed to establish relationships between side-to-side differences in running mechanics and side-to-side differences in clinical measures of pain, tendon structure, and calf muscle function.

Materials and methods 12 runners with Achilles tendinopathy were included (age: $44 \pm 11 \mathrm{y}$; height: $171 \pm 10 \mathrm{~cm}$; mass:70 $\pm 12 \mathrm{~kg}$; VISA-A score:71 \pm 10 ; current mileage: $40 \pm 29 \mathrm{~km} / \mathrm{wk}$ ). Participants completed a $7 \mathrm{~min}$ run at endurance pace $(2.9$ $\pm 0.3 \mathrm{~m} / \mathrm{s}$ ) on an instrumented treadmill with retroreflective markers affixed to their lower extremities. After a 6 min familiarization period, marker trajectories and ground reaction forces were sampled. Sagittal plane ankle joint moments and powers were calculated and a musculoskeletal model was used to estimate Achilles tendon loads. Pain-pressure threshold, tendon geometry, and calf muscle endurance were measured bilaterally with algometry, ultrasound imaging, and the heel-rise endurance test, respectively.

Results Side-to-side differences in pain-pressure threshold were significantly related to side-to-side differences in Achilles tendon loading rate $(\mathrm{r}=0.62 ; \mathrm{p}=0.03)$ and peak plantarflexion moment $(r=0.58, p=0.05)$. Side-to-side differences in peak eccentric ankle joint power were significantly related to sideto-side differences in tendon thickness $(r=0.59, \mathrm{p}=0.04)$ and cross-sectional area $(r=0.73 ; p=0.01)$. Side-to-side differences in calf muscle endurance was significantly related to Achilles tendon loading rate $(r=0.64 ; \mathrm{p}=0.03)$.

Conclusion Clinical measures of pain and calf muscle endurance relate to side-to-side differences in Achilles tendon loading rates during running, while tendon geometry relates to eccentric ankle joint power.

\section{THE ACHILLES TENDON TOTAL RUPTURE SCORE SHOULD BE USED WITH CAUTION THE FIRST 6 MONTHS AFTER INJURY}

\footnotetext{
${ }^{1,2}$ Maria Swennergren Hansen* ${ }^{*}{ }^{3}$ Katarina Nilsson Helander, ${ }^{3}$ Jón Karlsson, ${ }^{2}$ Kristoffe Weisskrichner Barfod. 'Physical Medicine and Rehabilitation Research-Copenhagen (PMRC); Department of Physical and Occupational Therapy, Copenhagen University Hospital Amager-Hvidovre, Denmark; ${ }^{2}$ Sports Orthopedic Research Center - Copenhagen (SORC-C), Department of Orthopedic Surgery, Copenhagen University Hospital Amager-Hvidovre, Denmark; ${ }^{3}$ Department of Orthopaedics, Sahlgrenska University Hospital, Sweden
}

\subsection{6/bjsports-2019-scandinavianabs.20}

Introduction The Achilles tendon Total Rupture Score (ATRS) is the most commonly used patient reported outcome in patients with an acute Achilles tendon rupture. The score 